\title{
BMJ Open A stepped-wedge cluster randomised controlled trial for evaluating rates of falls among inpatients in aged care rehabilitation units receiving tailored multimedia education in addition to usual care: a trial protocol
}

Anne-Marie Hill, ${ }^{1}$ Nicholas Waldron, ${ }^{2,3}$ Christopher Etherton-Beer, ${ }^{4,5}$ Steven M McPhail, ${ }^{6,7}$ Katharine Ingram, ${ }^{8}$ Leon Flicker, ${ }^{4,5}$ Terry $\mathrm{P}$ Haines ${ }^{9,10}$

To cite: Hill A-M, Waldron N, Etherton-Beer C, et al. A stepped-wedge cluster randomised controlled trial for evaluating rates of falls among inpatients in aged care rehabilitation units receiving tailored multimedia education in addition to usual care: a trial protocol. $B M J$ Open 2014;4:e004195. doi:10.1136/bmjopen-2013004195

- Prepublication history for this paper is available online. To view these files please visit the journal online (http://dx.doi.org/10.1136/ bmjopen-2013-004195).

Received 8 October 2013 Revised 7 December 2013 Accepted 11 December 2013

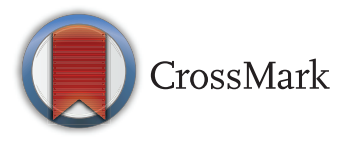

For numbered affiliations see end of article.

Correspondence to Dr Anne-Marie Hill; anne-marie.hill@nd.edu.au

\section{ABSTRACT}

Introduction: Falls are the most frequent adverse event reported in hospitals. Approximately $30 \%$ of in-hospital falls lead to an injury and up to $2 \%$ result in a fracture. A large randomised trial found that a trained health professional providing individualised falls prevention education to older inpatients reduced falls in a cognitively intact subgroup. This study aims to investigate whether this efficacious intervention can reduce falls and be clinically useful and costeffective when delivered in the real-life clinical environment.

Methods: A stepped-wedge cluster randomised trial will be used across eight subacute units (clusters) which will be randomised to one of four dates to start the intervention. Usual care on these units includes patient's screening, assessment and implementation of individualised falls prevention strategies, ongoing staff training and environmental strategies. Patients with better levels of cognition (Mini-Mental State Examination $>23 / 30$ ) will receive the individualised education from a trained health professional in addition to usual care while patient's feedback received during education sessions will be provided to unit staff. Unit staff will receive training to assist in intervention delivery and to enhance uptake of strategies by patients. Falls data will be collected by two methods: case note audit by research assistants and the hospital falls reporting system. Cluster-level data including patient's admissions, length of stay and diagnosis will be collected from hospital systems. Data will be analysed allowing for correlation of outcomes (clustering) within units. An economic analysis will be undertaken which includes an incremental costeffectiveness analysis.

Ethics and dissemination: The study was approved by The University of Notre Dame Australia Human Research Ethics Committee and local hospital ethics committees.

\section{Strengths and limitations of this study}

- This trial will evaluate whether a patient falls prevention education programme which showed efficacy in a randomised controlled trial can be clinically and economically effective when used in the real-world clinical environment.

- The stepped-wedge cluster design, which uses a large population of subacute care units, will provide robust evidence about the effectiveness of the intervention.

- The inclusion of an economic evaluation from the perspective of the health service provider will inform future implementation of this intervention.

- Hospital data coding of patient demographic and diagnostic information are collected within the central system and cannot feasibly be verified by the researchers.

- Research physiotherapists are limited in providing the projected number of hours per week required to deliver the education, consistent with hospital-based employment and therefore not all eligible patients are guaranteed to receive the intervention.

- Unanticipated systematic changes at sites may affect participation in the intervention or contaminate the trial results.

Results: The results will be disseminated through local site networks, and future funding and delivery of falls prevention programmes within WA Health will be informed. Results will also be disseminated through peer-reviewed publications and medical conferences.

Trial registration: The study is registered with the Australian New Zealand Clinical Trials registry (ACTRN12612000877886). 


\section{BACKGROUND}

Falls are the most common adverse event reported in hospitals, being between $20 \%$ and $30 \%$ of all incident reports. ${ }^{1}{ }^{2}$ Falls have potentially negative consequences for older inpatients. Approximately $30 \%$ of in-hospital falls result in physical injury, ${ }^{3-5}$ while fractures are a consequence in approximately $2 \% .{ }^{16}$ Composite falls rates reported across all hospital wards include areas such as surgical wards, where the incidence of falls is much lower than that on medical or rehabilitation wards. ${ }^{4}$ Subacute wards that admit older patients incur much higher rates of falls and higher proportions of patients falling, with rates as high as 20 falls $/ 1000$ patient-days. ${ }^{49}$

Patients who fall while in hospital increase health system costs. ${ }^{10} 11$ Some Australian data suggest that overall, hospital fallers stay approximately twice as long and have double the costs of non-fallers, ${ }^{11}$ while other Australian data indicate that the cost per fall is approximately equivalent between cognitively intact and cognitively impaired patients and that falling in hospital appears to affect length of stay (LOS) and subsequent costs arising on subacute wards more than acute wards. ${ }^{12}$

Four randomised trials that have investigated multifactorial falls prevention interventions in hospital ${ }^{9}{ }^{13-15}$ have been combined in a recent meta-analysis, which found that although falls can be reduced with targeted multifactorial interventions in patients who have longer LOS, the amount and intensity of each component required is unknown. ${ }^{16}$ Few studies have investigated the individual components that have been used in multifactorial interventions. A large cluster randomised controlled trial (RCT) tested an intervention designed to increase bed alarm use in hospitals, which increased alarm use, but had no statistically or clinically significant effect on fall-related events. ${ }^{17}$ Similarly, a large cluster trial which evaluated the use low-low beds in hospitals as a falls reduction strategy found that there was no significant difference in the rate of falls between intervention and control group wards, after the introduction of the low-low beds. ${ }^{18} \mathrm{~A}$ recent RCT which evaluated a falls prevention programme in hospitals observed that patients considered to be at high risk of falling who were provided with nurse-led education (as part of a targeted multiple intervention strategy) in addition to usual care had a lower falls rate $(0.4 \%)$ in comparison with patients who were provided with usual care $(1.5 \%) .{ }^{19}$ However, the low falls rates reported in that trial (for both control and intervention groups) and use of a multiple intervention strategy limit the ability to extrapolate the findings from that investigation to individualised patient education as a single intervention for the prevention of falls among inpatients in aged care rehabilitation units.

Recently, investigators from the present study published data from an RCT that is the largest trial to date of a single intervention (individualised patient education) designed to prevent falls in hospital. ${ }^{20}$ The intervention provided in the RCT demonstrated that an individualised patient education intervention provided by a trained health professional (known as the Safe Recovery programme), in addition to usual care, is effective for prevention of falls among older inpatients with intact cognition (adjusted HR 0.43 (95\% CI 0.24 to $0.78)$ ) but not for those with cognitive impairment. ${ }^{20}$

This trial enrolled individual patients who were randomised to intervention or control groups within the same ward. Thus, the intervention was not implemented as a ward-wide programme. Ward staff were not made aware of who was participating in the trial, were not involved in provision of the intervention and did not receive feedback gathered by the researchers who provided the Safe Recovery programme, which could have been used to improve usual care practice. A survey among hospital ward staff caring for trial participants indicated that staff could not identify who was in the intervention or control group beyond random chance. ${ }^{20}$ Although this reduced the risk of bias associated with this trial, it also reduced the ability to generalise trial results to real-life delivery of the intervention. Delivering this patient education in real-life clinical environments could reduce the effectiveness of the intervention as there may be a greater clinical heterogeneity of patients and less receptive patients receiving the intervention in real life than were recruited into the trial. Also, there may be a lower intervention fidelity, as the original efficacy trial had the intervention being provided by study investigators and research staff under their direct supervision. In real life, the study investigators would not be able to provide the intervention or supervise its provision directly. However, provision as a part of real-life clinical care could plausibly provide additional benefit as: (1) the educator who provides the Safe Recovery programme can liaise directly with unit staff as a part of the care team and engage in two-way dialogue about individual patients; (2) unit staff can help re-enforce messages provided during the education sessions (where appropriate) to individual patients; (3) information gathered from patients during the process of providing the Safe Recovery programme to improve broader quality improvement activities on the unit may enhance the usual care provided to all patients. It is unknown whether provision of this patient education intervention in a real-life clinical context will enhance or diminish the reduction in falls observed compared to the original efficacy RCT.

\section{Study aims}

This study aims to evaluate the effectiveness of falls prevention patient education, in real-life clinical environments, on rates of falls across participating subacute units. In addition, the study aims to evaluate the economic efficiency (incremental cost-effectiveness) of providing individualised patient education in this clinical context.

\section{METHODS}

\section{Design}

A stepped-wedge cluster design will be used (figure 1), with the duration of the trial being 50 weeks. The 


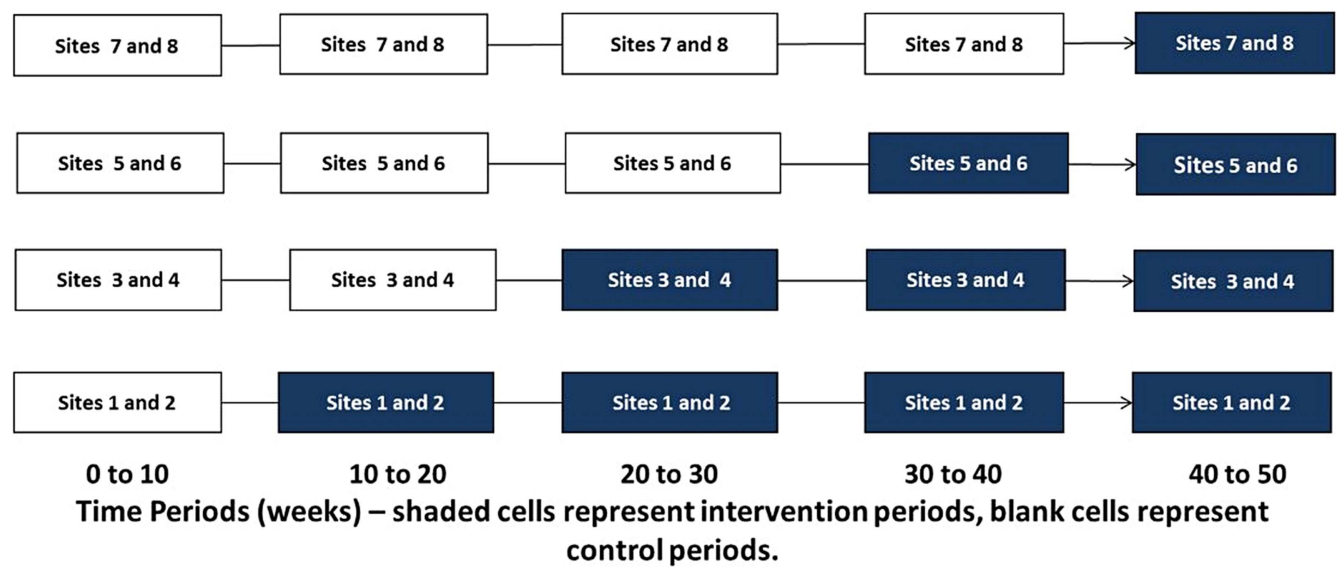

Figure 1 Stepped-wedge design.

'stepped-wedge' cluster randomised trial is a form of cross-over design with unidirectional cross-over (from control to experimental) but with randomisation of when each cluster undertakes this transition. ${ }^{21}{ }^{22}$ In the stepped-wedge design, there is a staggered roll-out of the intervention, where the time and hence the sequence of units (clusters) that will start the intervention at each period is determined by random allocation. The randomisation occurs before the start of the trial. All clusters start the trial in a control phase with no intervention being delivered at any site, then sequentially cross over from the control group to the intervention group, until all sites are receiving the intervention (figure 1). ${ }^{21}{ }^{22}$ Outcomes are measured on the study participants in all clusters at every time period, hence measurement of outcomes takes place at each step in the wedge; each cluster provides data points in the control and intervention conditions allowing each site to act as its own control.

\section{Participants and setting}

Eligible units are those who provide geriatric subacute care (rehabilitation or geriatric evaluation and management (GEM)) units. The units all operate within the Western Australian Department of Health (WA Health). There are eight units (total 251 beds at the start of trial expanding to 267 beds at trial end). The units vary in size consisting of 14, 17, 20, 20, 24 (expanding to 40 beds mid trial), 30, 36 and 90 beds. Patients are admitted to the units for evaluation and rehabilitation, with referrals predominantly from acute hospital wards and other hospitals and some referrals from the community or local emergency departments. The most frequent reason for admission is for functional decline due to conditions including falls, fractures, medical illness (including stroke, cardiac and pulmonary conditions) as well as following surgery (orthopaedic, general or neurosurgical). Other admissions aim to optimise care for patients with complex conditions such as Parkinson's disease and other neurological conditions. All patients admitted to these units with a rehabilitation episode of care will be prospectively screened on admission and where inclusion criteria are met will be given the education. The patient-level criteria for receiving the intervention are that the patient must be over 60 years old, cognitively intact as defined by Mini-Mental State Examination (MMSE) ${ }^{23}$ of greater than $23 / 30$ or an Abbreviated Mental Test Score (AMTS) ${ }^{24}$ of greater than $7 / 10$ and final confirmation by the treating clinical team that the patient is considered to be cognitively intact and likely to benefit from the intervention. Patients who speak English as a second language are able to receive the education and are invited to use an interpreter or family member to assist them to communicate. The patient's projected admission must be at least 3 days on the unit to receive the education; in the subacute units, the mean LOS across all sites is approximately 20 days so we envisage that nearly all cognitively intact patients will be eligible for inclusion in the trial. In the GEM units where LOS is between approximately 2 and 10 days across all GEM sites with a mean LOS of approximately 9 days, education will be provided to patients whose LOS is projected to be at least 3 days.

Patients will be excluded from receiving the education intervention if they receive a diagnosis of delirium. Recovery will need to be specifically documented by the registrar and the medical team will communicate with the research physiotherapist before the intervention is started (or continued). The Safe Recovery programme is not provided to patients with moderate or severe cognitive impairment (MMSE of less than 24/30 or AMTS of less than $8 / 10$ ). Patients who are permanently unable to mobilise and remain bedbound or are receiving palliative care will not receive the education.

\section{Randomisation and blinding}

Randomisation of the eight sites will be conducted 8 weeks prior to the start of study using a computergenerated number sequence, by an investigator not involved in assessment or delivery of the intervention 
(TPH). The allocation is then communicated to the chief investigator (A-MH). All sites are observed for 10 weeks with falls and patient-level data being collected; no site receives the intervention during the first 10 weeks of the study. After 10 weeks, two units begin the intervention and this procedure continues at 10 -week intervals, until all eight sites have crossed over into the intervention group (figure 1). The final phase of the trial when all sites are participating in the intervention finishes 10 weeks after the final sites begin receiving the intervention. Hence the trial period is 50 weeks. Units will be notified of the date that they will be entering the intervention phase of the trial prior to the start, as they require time to organise their participation. Training of unit staff for the trial and orientation of research staff is delivered in the 4 weeks prior to the start of intervention at each site. Patients and unit staff are not able to be blinded to receiving the intervention, as the intervention seeks to motivate patients and unit staff to become knowledgeable and empower them to be aware of the changes facilitated by the intervention. The research assistants who audit notes are not informed of the methodology of the trial including when sites enter the intervention arm of the trial, and they conduct audits in a different location to where the education is delivered. They audit patient's notes after discharge, including those patients who did not receive the education. However, it is possible these research assistants may inadvertently notice an entry in the medical record relating to an aspect of the Safe Recovery programme. Hospital data on LOS and falls events are collected routinely by hospital administrative staff who are not aware of which units are participating in this trial or which individual patients may have received education.

\section{Intervention}

Once a unit crosses over into the intervention phase, the research physiotherapist starts delivery of the patient education on the unit. All research physiotherapists have current experience in working with older people. The Safe Recovery programme that is provided as part of the intervention is identical to that which proved effective when tested in the earlier $\mathrm{RCT}^{20}{ }^{20}$ The programme provides tailored information for patients delivered using pedagogically sound adult education methods and was developed through earlier trials, ${ }^{15}{ }^{25}$ including being tested in a pilot work prior to the previous large efficacy RCT. $^{26}$ The programme is provided individually to each patient with between three and five occasions of service. The programme consists of two components: a multimedia education package (which consists of a digital video disc and a written workbook) and a series of individual follow-up sessions from the research physiotherapist. Simple key messages are theoretically grounded in the Health Belief Model ${ }^{27}$ For example, the programme includes discussion of " 3 simple steps for stopping falls: 1. Know if you need help to get up and walk around. 2. Ask for help. 3. Wait for help." The discussion alerts participants that they personally are at risk of falls during admission, gives them knowledge about the nature of falls (how and where falls usually occur in hospital), knowledge about the benefits of engaging in falls prevention activities, explains cues to action (when to actually engage in the falls prevention action) and facilitates self-efficacy to take action (such as asking staff for help).

The video materials are viewed individually by each patient using portable digital video playing equipment and external head phones and each patient is issued with a workbook. After the education is provided using the multimedia, patients receive between two and four individual follow-up sessions from the research physiotherapist. The physiotherapist has discretion over the duration of each session. The median (IQR) for all sessions is 25 (20-36) minutes. ${ }^{20}$ Each session follows a format of discussion that facilitates the patients to gain knowledge and set personal goals to engage in safe behaviours. Patients are assisted to write their goals in their workbook and goals are reviewed as the patient's mobility changes.

In this cluster trial, the Safe Recovery programme is delivered through the unit itself. The intervention extends the Safe Recovery programme to include staff and unit procedures, which allows the programme to be delivered as part of the ongoing clinical care on the ward. In the earlier efficacy RCT, ${ }^{20}$ staff were blinded to patient participation in the trial. No feedback to staff was provided by the educators and no feedback regarding unit environment was provided to unit managers. Qualitative research by the investigators indicated that older patients may engage in risk-taking that increases their risk of falls. ${ }^{28}$ Key factors that were found to influence risk-taking behaviour included willingness to ask for help, communication failure between and within older adults, informal care givers and health professionals and delayed provision of help. Importantly, health professionals and caregivers were identified as playing a central role in mitigating unnecessary risktaking, though some older adults appear more likely to take risks than others by virtue of their attitudes. ${ }^{28}$

Inclusion of the unit staff is facilitated in three key areas: (1) training programme for intervention delivery; (2) feedback from educator regarding patient's goals to staff on ward; and (3) information to unit about Safe Recovery programme. A formal online training programme has been developed and is provided to all educators (the research physiotherapists) over $6 \mathrm{~h}$. This training has been developed from further work by the research team ${ }^{28}$ and is delivered using adult education principles. ${ }^{29}$ The training facilitates the educators to understand the mechanism of hospital falls, conduct a falls threat appraisal with older patients and facilitate the patients to develop suitable strategies to reduce their falls risk. The unit allied health therapists receive the same online training programme which allows them to provide support for the educator on the unit regarding 
education of other staff about the Safe Recovery programme, follow-up with patients and assist patients to engage in their chosen strategies. The educators provide the patient's multidisciplinary team with feedback about the patient's goals after the patient has received the education, and assist patients to communicate with unit staff regarding their goals. A sticker is placed above the bedside which alerts the staff to identify which patients have received the programme. The goals which are written in the patient's workbook and kept by the bedside can be discussed with the patient by other members of staff who can assist the patient to engage in their chosen safety strategies. Finally, the educator provides information about the Safe Recovery programme to unit staff as a series of short presentations, and ongoing feedback to unit managers, alerting the unit to patients' general concerns regarding their ability to enact falls prevention strategies on the unit and patients' feedback regarding the unit environment.

\section{Programme fidelity}

Prior to the start of intervention at a site, the educator and unit-allied health therapists complete the online training programme. Unit staff, including the multidisciplinary team, receive an orientation and information session about the intervention. Patient's unit lists are checked by the educator and the unit-allied health therapist at the beginning of each session to ensure that all patients have been screened for inclusion in delivery of the education. Episodes of staff education and training are repeated for new staff on the unit.

\section{Outcome measures}

\section{Primary outcome measures}

The primary outcome measure will be patient falls during hospitalisation on a unit involved in the trial. Falls are defined as 'an event which results in a person coming to rest inadvertently on the ground or floor or other lower level. ${ }^{30}$ In-hospital falls data will be collected using two approaches: (1) data extracted from the paper-based hospital incident report system and (2) searching of patient's case notes on a rotating audit basis. Previous work conducted by the investigators and others has demonstrated that multiple approaches to falls data collection are required to gather valid data in the hospital setting. ${ }^{31}$ Falls data from patient's case notes will be collected by the research assistant. Falls will be classified as injurious if they result in bruising, laceration, dislocation, fracture, loss of consciousness or if the patient report persistent pain which is consistent with previous work in this field. ${ }^{20}$ Details of fall-related injuries and their treatment will also be captured. The number of patient admissions to the unit and the number of patient bed days (LOS for each patient) are collected routinely at each site. Participating sites will collate these data specifically for the time period of the study.

Demographic and clinical information are routinely collected for patients with rehabilitation care types and entered into the Quality of Care Register (a local health service administrative database). A raw output report will be provided by each participating unit for individual patients admitted during the trial period. These data include age, diagnosis, history of falls, admission living location and the Functional Independence Measure (FIM) which includes motor and cognitive items. ${ }^{32} 33$ Data are also collected on patients' comorbidities and complications interfering with the rehabilitation episode. Although cognitive status on admission, measured with either the $\mathrm{MMSE}^{23}$ or the AMTS, ${ }^{24}$ is not routinely reported, sites have been requested to enter these data in an additional field.

Process outcomes will be measured aiming to identify any gaps in implementation of and adherence to the education process. These include surveying patients to measure whether they have increased levels of knowledge, awareness and self-efficacy to engage in their falls prevention strategies after receiving the education and surveying patients to evaluate their satisfaction with the education programme. These outcomes will be collected by research physiotherapists who provide the patient education on the unit.

\section{Procedure}

Falls data are prospectively collected by the research assistant on an audit basis at all eight sites throughout the trial period of 50 weeks. Falls events are also entered into hospital incident report systems by unit staff as part of usual unit practice. All sites start the trial in the control phase. Starting at week 10 and subsequently at each 10-week interval, two sites enter the intervention phase of the trial according to their randomisation. The data collected at each site therefore act as an individual site's control conditions. All patients admitted to the unit are screened at admission and for those who meet the patient-level inclusion criteria the education is started in daytime hours from Monday through Friday, as soon as the patient is able to participate, that is, is medically stable. The research physiotherapist communicates with unit staff, including nursing and allied health staff, at each occasion of visiting the unit for updating individual patient-level strategies and any unit-level strategies noted to be required.

\section{Statistical analysis}

All analyses will be conducted on an intention-to-treat basis. The primary analyses will compare between intervention and control periods (1) the rate of falls (falls per 1000 patient days) using negative binomial regression, (2) the proportion of patients who experience one or more falls versus no falls using logistic regression and (3) the rate of falls resulting in injury using negative binomial regression. Each analysis will use patient-level data that will be clustered within 'unit'. Preplanned subgroup analyses of patients who are classified as being cognitively intact versus cognitively impaired will be conducted. Cognitive impairment will be classified using the MMSE 
cut-off of less than $24 / 30 .^{23}$ Best-subsets imputation will be used to calculate MMSE scores where missing, based on AMTS,${ }^{24}$ FIM cognitive subscale, ${ }^{32} 33$ age and diagnosis category (including diagnosis of depression). Analyses that examine an intervention-by- 'time since the start of the intervention on that unit' interaction effect will also be conducted to identify whether there was a cumulative unit-level effect of the intervention over time. This effect is plausible given the potential for the discussions held between patients and educators can be used to inform subsequent quality improvement efforts on the unit that will take time to manifest. Only data from patients admitted following the start of trial will be included, and the data will be censored at trial conclusion for patients remaining on the units after this point. Patients already admitted to the units at the point where the unit transitions from being a control unit to an intervention unit will have their data censored from the day prior to the start of transition. This is to avoid contamination of data analyses by patients who are exposed to control and intervention periods on their unit. All of these analyses will be adjusted for covariates of the FIM (motor and cognitive score),$^{32}$ the number of comorbidities, age, gender and the rate of falls for the same time period from the previous year at the research locations.

The final primary analysis will be of differences in LOS on the unit between intervention and control periods. This analysis will only include data from patients who were admitted and discharged during the unit's control period or intervention period. Patients who had their data censored at the conclusion of the control or intervention periods will not be included in this analysis of LOS. Linear regression analysis will be employed with patients clustered by 'site' with the dependent variable being examined for $\log$ (or other) transformation to improve the model fit (log transformations are often necessary given frequently observed skewed distributions of LOS data).

Secondary analyses will also be conducted comparing fall outcomes and LOS within individual units. An intervention-by-unit interaction effect will be investigated and random-effects metaregression of unit-level analyses will be undertaken to calculate the $\mathrm{I}^{2}$ statistic, which describes the percentage of total variation across sites that is due to heterogeneity rather than chance. ${ }^{34} \mathrm{~A}$ feature of the stepped wedge is that each unit provides control and intervention period data; this provides an opportunity to investigate the homogeneity of intervention effect using this method not readily afforded by other trial designs.

\section{Power calculation}

We have eight sites with an average of approximately 26 beds per unit and the observation period is 50 weeks. If we assume that the intervention will have an effect on the incidence rate of falls of 0.7 (30\% relative reduction) and we use a two-tailed $\alpha$ of 0.05 , and power of 0.80 , and if we assume a rate of falls of $10 / 1000$ bed days and a mean LOS of 20 days per patient, then we need 1485 patients in total in a standard RCT. However, the stepped wedge is a form of cluster randomised trial; therefore, we multiply the sample size by the design effect which $=1+(\mathrm{m}-1) \times \mathrm{ICC}$, where $\mathrm{m}=$ average cluster size and ICC is the intraclass correlation coefficient. Since we have eight sites with an average of approximately 26 beds per unit, if the mean LOS is 20 days then each unit would contribute an average of 468 patients over the year. The ICC from a previous falls prevention cluster-randomised trial from the rehabilitation wards ${ }^{13}$ was 0.002. Thus, the design effect is 1.6. Therefore, the total sample size required is 2409 . If each site contributes 468 patients over the year, we will have 3744 patients in the trial which is greater than the 2409 required for a 0.7 effect size to have $80 \%$ power.

\section{Economic analysis}

Two forms of economic evaluation will be undertaken for this project: (1) an incremental cost-effectiveness analysis based on primary trial data; (2) a net benefit analysis based on modelling a broader roll-out of this programme using data arising from this trial.

An incremental cost-effectiveness analysis will identify the 'cost per fall prevented' from implementation of the Safe Recovery programme. This analysis will be taken from the 'health service provider' (WA Health) stakeholder perspective. The denominator in this ratio will be the difference in the rate of patient falls during the intervention period compared with the control period. The numerator of this ratio will be driven by costs associated with training staff to provide the Safe Recovery programme, implementing changes to usual care arising from feedback received while delivering the Safe Recovery programme and changes in LOS and hospital services provided to patients that are attributable to falls.

The costs of training staff to provide the Safe Recovery programme will be taken using market rates for this training (\$A440 per participant inclusive of goods and service taxes) even though it is being provided in-kind for the present project. It is anticipated that this training will also take place during work hours for unit staff and that their roles will require 'back-fill' while undertaking this training. Thus, hourly wage rates with on-costs will be included as a part of training costs. These training costs will be divided by the mean number of patients seen by each staff member during the trial (though this is likely to be a conservative estimate of this cost given the short-term nature of the trial and the ability for these staff to provide the programme to patients after the trial has concluded).

Costs of providing the Safe Recovery programme will be derived from payments made to employ research physiotherapists in this capacity and the cost of purchasing digital video playing equipment and printing costs for patient's workbooks. The cost of employing research physiotherapists to provide the Safe Recovery programme will include the hourly wage rate and additional 
on-cost calculations to cover additional employment expenses such as superannuation and annual leave entitlements. Research physiotherapists employed in this capacity record all time spent in activities related to delivering the Safe Recovery programme. Hence, the information collected by personnel providing the Safe Recovery programme will be able to be used to inform sensitivity analyses in this regard. The time of staff who do not provide the Safe Recovery programme will also be modelled in this analysis based on data collected during the trial. This will be estimated for when they are involved in activities, where they are discussing feedback received by the Safe Recovery programme provider, time spent forming a response to this feedback and enacting changes in practice in light of this feedback.

Costs associated with serious injury (such as hip fracture) with extended additional hospitalisation on acute wards and possible surgical procedures will be able to be followed up on a case-by-case basis since injuries as serious as this tend to occur in only approximately $2 \%$ of falls. ${ }^{6}{ }^{20}$ Changes in LOS will be directly measured during this trial and valued using contemporary funding models that regulate payments to WA hospitals to ensure that the findings have direct applicability to health services. ${ }^{35}$ Other costs directly attributable to falls such as the provision of analgesic therapies or taking neurological observations have been found to have a much smaller impact on total cost per fall estimates ${ }^{12}$ and thus will not be directly measured in this trial.

Once all these relevant costs have been counted and valued, the incremental cost-effectiveness ratio will be calculated. Bootstrap resampling will be employed to construct a 95\% confidence ellipse around this costeffectiveness estimate and to perform acceptability curve analysis which will inform stakeholders as to the probability that the programme is less costly and more clinically beneficial to provide compared with usual care. Sensitivity analyses will be performed varying key cost input variables within clinically plausible ranges to identify areas that the main finding of the economic evaluation is sensitive to.

A net benefit analysis of broader roll-out of this programme will then be performed using a decision tree analysis approach. Potential roll-out to a range of hospitals (metropolitan, regional, remote) will be modelled using a net-benefit approach. Net benefit analyses calculate a dollar value of cost versus benefit to the stakeholder (in this case, WA Health) so that policymakers can accurately estimate the costs or savings they are likely to encounter with broader roll-out of this programme. These models will be largely based on data collected during the trial, though inclusion of data from other published sources ${ }^{10}{ }^{12}$ and administrative databases, such as the existing rate of falls of other WA Health hospital sites, will also be undertaken. Key variables to be included in the decision tree analysis will be the rate of falls on wards to be targeted with future roll-out (drawn from WA Health data), the effectiveness of the intervention in preventing falls (drawn from the trial) and the cost per faller versus non-faller (drawn from the trial and from previous research). ${ }^{10} 12$

\section{DISCUSSION}

This study is the world's first effectiveness study of a falls prevention intervention in the hospital setting to build on the findings of an earlier efficacy trial. It is important that interventions developed and tested in controlled efficacy study environments are tested in real life, effectiveness studies so the generalisability of the original efficacy study findings can be known. As such, this trial will make a vital contribution to the evidence base for the prevention of falls in hospitals.

The Safe Recovery programme, that has demonstrated effectiveness in a clinical trial with individual randomisation, provided older patients with clear simple message and an action plan to use while in hospital. ${ }^{20}$ However, delivering this intervention to the whole unit in a real-life context may increase or decrease its effect and costefficiency. Qualitative studies have found that older patients who could recall receiving ward falls prevention information reported that they needed more consistent reminders, assistance and instructions. ${ }^{36} 37$ Other qualitative research has found that a participatory approach involving frontline staff, managers, researchers, staff, family members and patients can be feasible and helpful for developing strategies to prevent falls among older people in a geriatric rehabilitation setting. ${ }^{38}$ This intervention aims to provide older patients with high levels of knowledge, confidence and motivation to reduce their falls risk, and also to empower staff to ensure that patients understand the safety instructions and the procedures on the unit.

The unit-level implementation of this intervention using a randomised stepped-wedge design is likely to provide high-quality data to indicate whether this intervention can prevent falls, reduce LOS and save money from the perspective of health service providers. This design also allows for a novel investigation of intervention homogeneity by conducting a meta-analysis of unit-level data so that the $\mathrm{I}^{2}$ statistic can be calculated. The inclusion of an economic evaluation from the perspective of the health service provider, to be conducted from data collected in this study and other sources, may be considered a strength of this investigation for informing future implementation of this intervention. It is anticipated that this investigation will yield findings that indicate whether unit-level implementation of this intervention is likely to be a dominant strategy (saves money and improves outcomes) and whether or not this is a cost-effective strategy. The outcome of the economic evaluation will likely be dependent on whether the intervention not only reduces falls rates, but also influences patient's LOS. Changes in LOS have been found to be the key driver of costs in hospital-based falls prevention programmes. ${ }^{10-12}$ Although difficulty exists in attributing causation with regard to falling and LOS, it is likely that 
capacity exists to reduce LOS through preventing falls events in hospital.

The main limitation of the trial is that all collected hospital data are collected routinely in a central system and it may not be possible to find missing data or determine inaccurate coding. In addition, the research physiotherapists are not placed onto each unit in a full time capacity but are limited by the projected number of hours required to deliver the education. Therefore, not all eligible patients are guaranteed to receive the intervention. The unidirectional nature of the cross-over (from control to intervention) within the stepped wedge may mean that factors external to the trial (eg, the start of other interventions such as universal prescription of vitamin $\mathrm{D}$, systematic changes to staffing profile, activities or patient casemix) have the potential to contaminate the trial results if they arise.

\section{CONCLUSION}

This stepped-wedge cluster randomised trial will generate translational data regarding the clinical use and costeffectiveness of individualised patient falls prevention education when applied in a real-time clinical environment. The results will inform policymakers and ward-level approaches to falls prevention programmes, and if efficacy is maintained, or enhanced, in the clinical roll-out, will provide specific guidance in how to reduce falls in subacute units.

\section{Author affiliations \\ ${ }^{1}$ School of Physiotherapy, The University of Notre Dame Australia, Fremantle, Western Australia, Australia \\ ${ }^{2}$ Department of Rehabilitation and Aged Care, Armadale Kelmscott Memorial Hospital, Perth, Western Australia, Australia \\ ${ }^{3}$ Health Strategy and Networks, Strategic System, Policy and Planning, Department of Health, Government of Western Australia, Perth, Western Australia, Australia \\ ${ }^{4}$ School of Medicine and Pharmacology, WA Centre for Health \& Ageing CMR, University of Western Australia, Perth, Western Australia, Australia \\ ${ }^{5}$ Royal Perth Hospital, Perth, Western Australia, Australia \\ ${ }^{6}$ Institute of Health and Biomedical Innovation and School of Public Health \& Social Work, Queensland University of Technology, Kelvin Grove, Queensland, Australia \\ ${ }^{7}$ Centre for Functioning and Health Research, Metro South Health, Brisbane, Australia \\ ${ }^{8}$ Department of Rehabilitation and Aged Care, Sir Charles Gairdner Hospital, Perth, Western Australia, Australia \\ ${ }^{9}$ Physiotherapy Department, Monash University, Frankston, Victoria, Australia \\ ${ }^{10}$ Allied Health Research Unit, Monash Health, Cheltenham, Victoria, Australia}

Contributors A-MH contributed to study conception, design, trial management, original manuscript drafting, appraisal and editing. TPH and SMM contributed to the study conception, design, original manuscript drafting, appraisal and editing. NW, CE-B, KI and LF contributed to study conception, design, trial management, manuscript appraisal and editing. All authors read and approved the final manuscript.

Funding This work was supported by the Western Australian State Health Research Advisory Council and the Department of Health, Western Australia as part of the Research Translation Projects programme.

Competing interests TPH is the Director of Hospital Falls Prevention Solutions Pty Ltd. This company provides training for provision of the Safe
Recovery programme. As such, TPH has a direct financial interest in the outcomes of this research. TPH has been involved in the conception of this research, the selection of the research design, the analysis approach, the design of the economic evaluation and the drafting of this manuscript. He is providing training in the Safe Recovery programme to project personnel at no cost to the project. He has no involvement in the extraction of data or the analysis of data beyond providing guidance as to appropriate methods for conducting the analysis at the stage of writing the trial protocol. He has no agreement or ability that restricts the ability of other investigators to publish the trial results. TPH has provided expert witness testimony for Minter Ellison Law Firm on the subject of prevention of falls in hospital and has received speakers' fees to talk on the subject of the prevention of falls at conferences. A-MH and SMM are supported by the National Health and Medical Research Council (of Australia) fellowships. TPH is supported by a National Health and Medical Research Council (of Australia) Career Development award.

Ethics approval The University of Notre Dame Australia HREC, Sir Charles Gairdner Hospital Osborne Park group HREC, reciprocal approval South Metropolitan Health Service HREC, Royal Perth Hospital HREC.

Provenance and peer review Not commissioned; externally peer reviewed.

Open Access This is an Open Access article distributed in accordance with the Creative Commons Attribution Non Commercial (CC BY-NC 3.0) license, which permits others to distribute, remix, adapt, build upon this work noncommercially, and license their derivative works on different terms, provided the original work is properly cited and the use is non-commercial. See: http:// creativecommons.org/licenses/by-nc/3.0/

\section{REFERENCES}

1. Healey F, Scobie S, Oliver D, et al. Falls in English and Welsh hospitals: a national observational study based on retrospective analysis of 12 months of patient safety incident reports. Qual Saf Health Care 2008;17:424-30.

2. Healthcare in focus how well does NSW perform? An international comparison. Bureau of Health Information, 2011. http://www.bhi.nsw. gov.au/publications/annual_performance_report_series/healthcare_ in focus 2011 (accessed 30 Sep 2013)

3. Hill AM, Hoffmann T, Hill K, et al. Measuring falls events in acute hospitals-a comparison of three reporting methods to identify missing data in the hospital reporting system. J Am Geriatr Soc 2010;58:1347-52

4. Schwendimann $\mathrm{R}$, Buhler $\mathrm{H}$, De Geest $\mathrm{S}$, et al. Characteristics of hospital inpatient falls across clinical departments. Gerontology 2008;54:342-8.

5. Hitcho EB, Krauss MJ, Birge S, et al. Characteristics and circumstances of falls in a hospital setting. A prospective analysis. J Gen Intern Med 2004;19:732-9.

6. Nadkarni JB, lyengar KP, Dussa C, et al. Orthopaedic injuries following falls by hospital in-patients. Gerontology 2005;51:329-33.

7. Milisen K, Staelens N, Schwendimann R, et al. Fall prediction in inpatients by bedside nurses using the St. Thomas's risk assessment tool in falling elderly inpatients (STRATIFY) instrument: a multicenter study. J Am Geriatr Soc 2007;55:725-33.

8. Haines T, Kuys SS, Morrison G, et al. Balance impairment not predictive of falls in geriatric rehabilitation wards. J Gerontol A Biol Sci Med Sci 2008;63:523-8.

9. Healey F, Monro A, Cockram A, et al. Using targeted risk factor reduction to prevent falls in older in-patients: a randomised controlled trial. Age Ageing 2004;33:390-5.

10. Bates DW, Pruess K, Souney $P$, et al. Serious falls in hospitalized patients: correlates and resource utilization. $\mathrm{Am} \mathrm{J}$ Med 1995;99:137-43.

11. Hill KD, Vu M, Walsh W. Falls in the acute hospital setting-impact on resource utilisation. Aust Health Rev 2007;31:471-7.

12. Haines TP, Hill AM, Hill KD, et al. Cost effectiveness of patient education for the prevention of falls in hospital: economic evaluation from a randomized controlled trial. BMC Med 2013;11:135.

13. Cumming RG, Sherrington C, Lord SR, et al. Cluster randomised trial of a targeted multifactorial intervention to prevent falls among older people in hospital. BMJ 2008;336:758-60.

14. Stenvall $M$, Olofsson $B$, Lundstrom $M$, et al. A multidisciplinary, multifactorial intervention program reduces postoperative falls and injuries after femoral neck fracture. Osteoporos Int 2007;18:167-75.

15. Haines TP, Bennell $\mathrm{KL}$, Osborne $\mathrm{RH}$, et al. Effectiveness of targeted falls prevention programme in subacute hospital setting: randomised controlled trial. BMJ 2004;328:676. 
16. Cameron ID, Gillespie LD, Robertson MC, et al. Interventions for preventing falls in older people in care facilities and hospitals. Cochrane Database Syst Rev 2012;(12):CD005465.

17. Shorr RI, Chandler AM, Mion LC, et al. Effects of an intervention to increase bed alarm use to prevent falls in hospitalized patients: a cluster randomized trial. Ann Intern Med 2012;157:692-9.

18. Haines TP, Bell RA, Varghese PN. Pragmatic, cluster randomized trial of a policy to introduce low-low beds to hospital wards for the prevention of falls and fall injuries. J Am Geriatr Soc 2010;58:435-41.

19. Ang E, Mordiffi SZ, Wong HB. Evaluating the use of a targeted multiple intervention strategy in reducing patient falls in an acute care hospital: a randomized controlled trial. J Adv Nurs 2011;67:1984-92.

20. Haines TP, Hill AM, Hill KD, et al. Patient education to prevent falls among older hospital inpatients: a randomized controlled trial. Arch Intern Med. 2010;171:516-24.

21. Brown $\mathrm{C}$, Lilford R. The stepped wedge trial design: a systematic review. BMC Med Res Methodol 2006;6:54

22. Hussey MA, Hughes JP. Design and analysis of stepped wedge cluster randomized trials. Contemp Clin Trials 2007;28:182-91.

23. Folstein MF, Folstein SE, McHugh PR. "Mini-mental state". A practical method for grading the cognitive state of patients for the clinician. J Psychiatr Res 1975;12:189-98.

24. Hodkinson HM. Evaluation of a mental test score for assessment of mental impairment in the elderly. Age Ageing 1972;1:233-8.

25. Haines TP, Hill KD, Bennell KL, et al. Patient education to prevent falls in subacute care. Clin Rehabil 2006;20:970-9.

26. Hill AM, McPhail S, Hoffmann $\mathrm{T}$, et al. A randomized trial comparing digital video disc with written delivery of falls prevention education for older patients in hospital. J Am Geriatr Soc 2009;57:1458-63.

27. Janz NK, Becker MH. The Health Belief Model: a decade later. Health Educ Q 1984;11:1-47.
28. Haines TP, Lee DC, O'Connell B, et al. Why do hospitalized older adults take risks that may lead to falls? Health Expect 2012. Published Online First: 29 November 2012; doi:10.1111/hex.12026

29. Merriam SB, Caffarella RS, Baumgartner LM. Learning in adulthood: a comprehensive guide. 3rd edn. San Francisco: Jossey-Bass, 2007:271-317.

30. WHO. 2010. http://www.who.int/violence_injury_prevention/other_ injury/falls/en/index.html (accessed 30 Sept 2013).

31. Shorr RI, Mion LC, Chandler AM, et al. Improving the capture of fall events in hospitals: combining a service for evaluating inpatient falls with an incident report system. J Am Geriatr Soc 2008;56:701-4.

32. Granger CV, Hamilton BB, Linacre JM, et al. Performance profiles of the functional independence measure. Am J Phys Med Rehabil 1993;72:84-9.

33. Granger CV, Markello SJ, Graham JE, et al. The uniform data system for medical rehabilitation: report of patients with stroke discharged from comprehensive medical programs in 2000-2007. Am J Phys Med Rehabil 2009;88:961-72.

34. Higgins JP, Thompson SG, Deeks JJ, et al. Measuring inconsistency in meta-analyses. BMJ 2003;327:557-60.

35. Activity based funding and management (2013-2014). Department of Health, Western Australia performance activity and quality division. http://www.health.wa.gov.au/activity/home/index.cfm (accessed 30 Sep 2013).

36. Tzeng HM, Yin CY. Perspectives of recently discharged patients on hospital fall-prevention programs. J Nurs Care Qual 2009;24: 42-9.

37. Carroll DL, Dykes PC, Hurley AC. Patients' perspectives of falling while in an acute care hospital and suggestions for prevention. App Nurs Res 2010;23:238-41.

38. Vieira ER, Berean C, Paches D, et al. Reducing falls among geriatric rehabilitation patients: a controlled clinical trial. Clin Rehabil 2013;27:325-35. 